\title{
Exploring mechanisms inhibiting nuclear fusion
}

\author{
M. Evers*, D. J. Hinde, M. Dasgupta \\ Department of Nuclear Physics, Research School of Physics and Engineering, The Australian \\ National University, Canberra, ACT 0200, Australia \\ Email: maurits.everseanu.edu.au
}

\begin{abstract}
Understanding the mechanisms leading to the inhibition of fusion seen at sub- and above-barrier energies has been one of the prime questions in nuclear physics. The complementary process to fusion is scattering, and an analysis of the scattered flux in heavy-ion collisions may give insight into answering this question. An analysis of the projectile-like fragments detected at backward angles in the reactions ${ }^{16} \mathrm{O}+{ }^{208} \mathrm{~Pb}$ and ${ }^{32} \mathrm{~S}+{ }^{208} \mathrm{~Pb}$ at energies below the fusion barrier is presented. Excitation functions corresponding to nucleon transfer with $\Delta Z=1$ and $\Delta Z=2$ were extracted, indicating surprisingly large absolute transfer probabilities already at sub-barrier energies. Excitation energies in the projectile-like fragments up to $\sim 15 \mathrm{MeV}$ for the ${ }^{16} \mathrm{O}$ and $\sim 25 \mathrm{MeV}$ for ${ }^{32} \mathrm{~S}$-induced reactions show the population of highly excited states in the residual nuclei, and indicate substantial dissipation of kinetic energy into nucleonic degrees of freedom. A new phenomenological framework explores the effect of energy dissipation through these highly inelastic (large excitation energies) and complex (few-nucleon transfer) processes on the probability for fusion in the reaction ${ }^{16} \mathrm{O}+{ }^{208} \mathrm{~Pb}$. Calculations combining both couplings to low-lying excited states within the coherent coupled channels framework and energy dissipation show that the suppression of fusion at energies above the barrier in this reaction can be explained within the new framework. Results furthermore suggest that in reactions relevant to astrophysical scenarios such as ${ }^{12} \mathrm{C}+{ }^{12} \mathrm{C}$, fusion hindrance may not be present.
\end{abstract}

XII International Symposium on Nuclei in the Cosmos,

August 5-12, 2012

Cairns, Australia

${ }^{*}$ Speaker. 


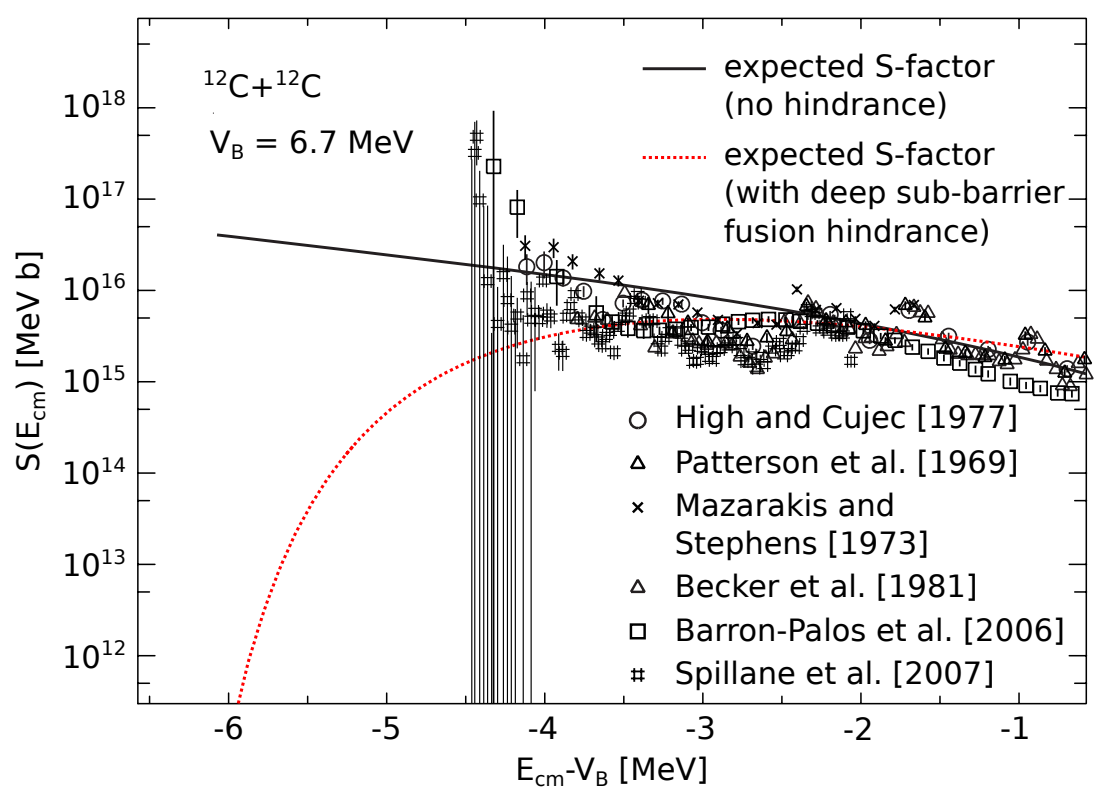

Figure 1: $S$-factor for the reaction ${ }^{12} \mathrm{C}+{ }^{12} \mathrm{C}$. Fusion cross section measurements are from Refs. [8, 9, 10, $11,12,13]$. The solid (black) and dotted (red) curves schematically illustrate the effect of deep sub-barrier fusion hindrance on calculated $S$-factors. The fusion barrier energy $V_{B}$ was taken from Ref. [14].

\section{Introduction}

Nuclear fusion is the process responsible for energy production in stars and for the formation of elements heavier than hydrogen in the early Universe. One critical parameter that determines the probability for fusion to occur is the energy height of the fusion barrier, that arises due to the interplay between the repulsive Coulomb and attractive nuclear potential. At energies well below and close to the fusion barrier, fusion occurs through quantum-mechanical tunnelling of the projectile nucleus through the barrier. Furthermore sub-barrier fusion as well as its (more likely) complementary scattering process are affected by the internal structure of the collision partners. The coupled reaction channels formalism which considers the colliding nuclei to be in a quantum-mechanical coherent superposition of intrinsic states has proven extremely successful in the description of both fusion and scattering at sub- and near-barrier energies [1, 2, 3].

However, at deep sub-barrier energies (e.g. $\sim 5 \mathrm{MeV}$ below the fusion barrier energy in the reaction ${ }^{16} \mathrm{O}+{ }^{208} \mathrm{~Pb}$ ) measured fusion cross sections fall below those predicted by coupled reaction channels calculations using standard Woods-Saxon potentials. This deep sub-barrier fusion hindrance has been observed in a range of different reactions [4, 5, 6, 7]. A major question in nuclear physics is to explain the physical mechanism(s) causing this suppression of the tunnelling probability.

This question is one of particular importance for astrophysical scenarios, as extrapolations of fusion probabilities to energies typical for astrophysical processes can show large variations for different phenomenological models [15, 16, 17]. For example, measurements of fusion cross sections $\sigma_{\text {fus }}$ for the reaction ${ }^{12} \mathrm{C}+{ }^{12} \mathrm{C}[8,9,10,11,12,13]$ are shown in Fig. 1 in terms of the corresponding $S$-factors $S=E_{c m} \sigma_{\text {fus }} \exp 2 \pi \eta$, where $E_{c m}$ is the centre-of-mass energy and $\eta=Z_{1} Z_{2} e^{2} \mu /\left(\hbar^{2} k\right)$ is 
the Sommerfeld parameter describing the strength of the Coulomb interaction between two nuclei with atomic numbers $Z_{1}, Z_{2}$ and reduced mass $\mu$. Direct measurements of the fusion probabilities at energies far below the barrier are often difficult due to the small fusion cross sections and competition with other more dominant reaction processes. In the absence of experimental measurements, extrapolations of the $S$-factor to energies corresponding to particular astrophysical scenarios are used to calculate reaction rates. In Fig. 1 the solid black and dotted red curves schematically illustrate the effect of deep sub-barrier fusion hindrance $[18,19]$ on the calculated $S$-factor for the reaction ${ }^{12} \mathrm{C}+{ }^{12} \mathrm{C}$. In the absence of any hindrance, the $S$-factor slowly increases as the energy decreases, whilst if fusion hindrance is present, the $S$-factor reaches a maximum before decreasing again with decreasing interaction energy. Depending on the degree of deep sub-barrier fusion hindrance in model calculations, reaction rates based on extrapolated (calculated) $S$-factors may give very different results [17].

At energies above the fusion barrier, measured fusion cross sections also significantly fall below standard coupled-channels calculations $[6,20]$ using a standard Woods-Saxon parametrization for the nuclear potential. A detailed analysis of this above-barrier fusion suppression for different reactions shows an increase in the amount of suppression with the charge product of the colliding nuclei [20]. The increase of above-barrier fusion suppression is related to an increasing dissipation of kinetic energy into nucleonic degrees of freedom, known as deep inelastic collisions (DIC) $[20,21]$. This becomes important with increasing matter overlap at energies near and above the fusion barrier. Transfer processes that lead to high excitation energies in the residual nuclei were suggested $[20,22]$ as a key to understanding above-barrier fusion suppression through the onset of irreversible dissipative processes.

In this paper recent results are discussed suggesting that mechanisms used to explain abovebarrier fusion suppression may also be (in part) responsible for the suppression of fusion through tunnelling at deep sub-barrier energies. Measurements of the reflected flux in the reactions of ${ }^{16} \mathrm{O}$ and ${ }^{32} \mathrm{~S}$ with ${ }^{208} \mathrm{~Pb}$ indicate that transfer processes in nuclear collisions play an important role already at energies well below the fusion barrier and lead to the dissipation of kinetic energy through the population of highly excited states in the residual nuclei. A new probabilistic framework for the description of fusion has been developed which takes into account the irreversible dissipation of kinetic energy, and is in excellent agreement with measured above-barrier fusion cross sections. Results support a strong link between fusion suppression (observed both at deep sub-barrier and above-barrier energies) and irreversible energy dissipation through transfer leading to highly excited states. Since the density of excited states at a fixed excitation energy is much lower for lighter nuclei than for heavier nuclei, this suggests that deep sub-barrier fusion suppression in astrophysically relevant processes ( such as ${ }^{12} \mathrm{C}+{ }^{12} \mathrm{C}$ ) may not be present, thus affecting reaction rates through extrapolated $S$-factors.

\section{Measurements}

All measurements were done with the 14UD electrostatic accelerator of the Heavy-Ion Accelerator Facility at the Australian National University (ANU), using beams of ${ }^{16} \mathrm{O}$ and ${ }^{32} \mathrm{~S}$ incident on a ${ }^{208} \mathrm{PbS}$ target with a thickness of $100 \mu \mathrm{g} / \mathrm{cm}^{2}$, evaporated onto a $15 \mu \mathrm{g} / \mathrm{cm}^{2} \mathrm{C}$ backing. A $\Delta E-E$ detector telescope consisting of a propane gas filled ionization chamber and a Si detec- 


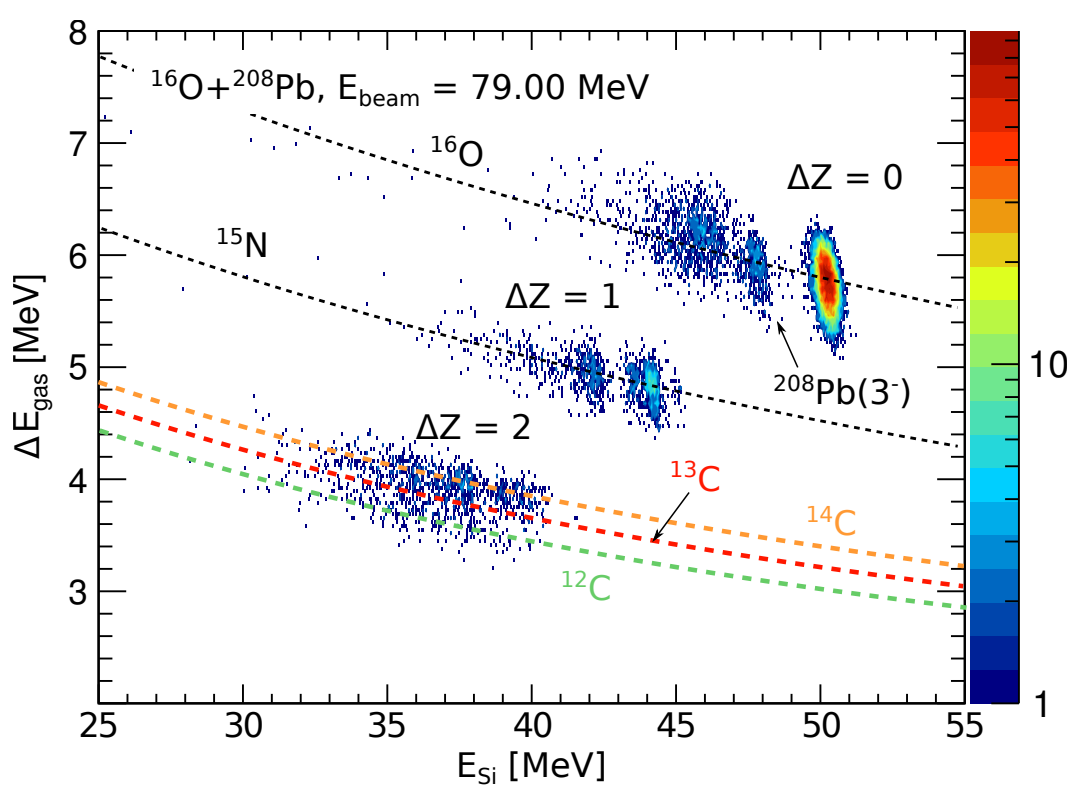

Figure 2: Typical $\Delta E-E$ spectrum for the reaction ${ }^{16} \mathrm{O}+{ }^{208} \mathrm{~Pb}$ at the indicated beam energy, corresponding to $E_{c . m .} / V_{B}=0.98$. Events corresponding to the transfer of $\Delta Z=0,1$ and 2 units of charge are labelled. Calculated energy loss curves for ${ }^{16} \mathrm{O},{ }^{15} \mathrm{~N}$ and ${ }^{12,13,14} \mathrm{C}$ are shown by the dashed curves.

tor located at a backward angle of $\theta_{\mathrm{lab}}=162^{\circ}$ was used to record the energy $E_{\mathrm{Si}}$ and energy loss $\Delta E_{\text {gas }}$ of the back-scattered projectile-like fragments (PLFs). Two Si monitors positioned at $\pm 30^{\circ}$ were used to normalize the back-scattered events to the Rutherford cross section. A typical two dimensional spectrum for a measurement of the reaction ${ }^{16} \mathrm{O}+{ }^{208} \mathrm{~Pb}$ at a beam energy corresponding to $E_{c . m .} / V_{B}=0.98$ is shown in Fig. 2. The three distinct regions correspond to oxygen, nitrogen and carbon PLFs, which are associated with the transfer of $\Delta Z=0,1$ and 2 units of charge. The main events at $E_{\mathrm{Si}} \sim 50 \mathrm{MeV}$ are due to elastically scattered ${ }^{16} \mathrm{O}$ particles, the smaller peak at $E_{\mathrm{Si}} \sim 48 \mathrm{MeV}$ is associated with the excitation of the lowest $3^{-}$excited state in ${ }^{208} \mathrm{~Pb}$ at an excitation energy of $2.615 \mathrm{MeV}$. Events resulting from the transfer of three or more charged nucleons $(\Delta Z \geq 3)$ are not observed for measurements at sub-barrier energies. Spectra for measurements of the PLFs following the reaction ${ }^{32} \mathrm{~S}+{ }^{208} \mathrm{~Pb}$ show similar features.

\section{Transfer probabilities}

Transfer probabilities for processes with different $\Delta Z$ were extracted by gating on the particular region of interest in the $\Delta E-E$ spectra, and normalizing the number of events to the total number of counts in the two forward angle monitor detectors. Overall normalization of the probabilities was obtained from measurements of the total quasi-elastic scattering yields at energies well below the barrier energy, following the procedure detailed in Ref. [3]. Isotopic identification and separation of the different back-scattered PLFs was possible using a combination of $Q$-value considerations for the different reaction channels and calculated energy loss curves for the different PLFs as shown in Fig. 2 for the reaction ${ }^{16} \mathrm{O}+{ }^{208} \mathrm{~Pb}$. Energy loss calculations were carried out using the code STROP3, which uses stopping power values from Ref. [25]. By using beams of ${ }^{12,13} \mathrm{C}$ in the same 


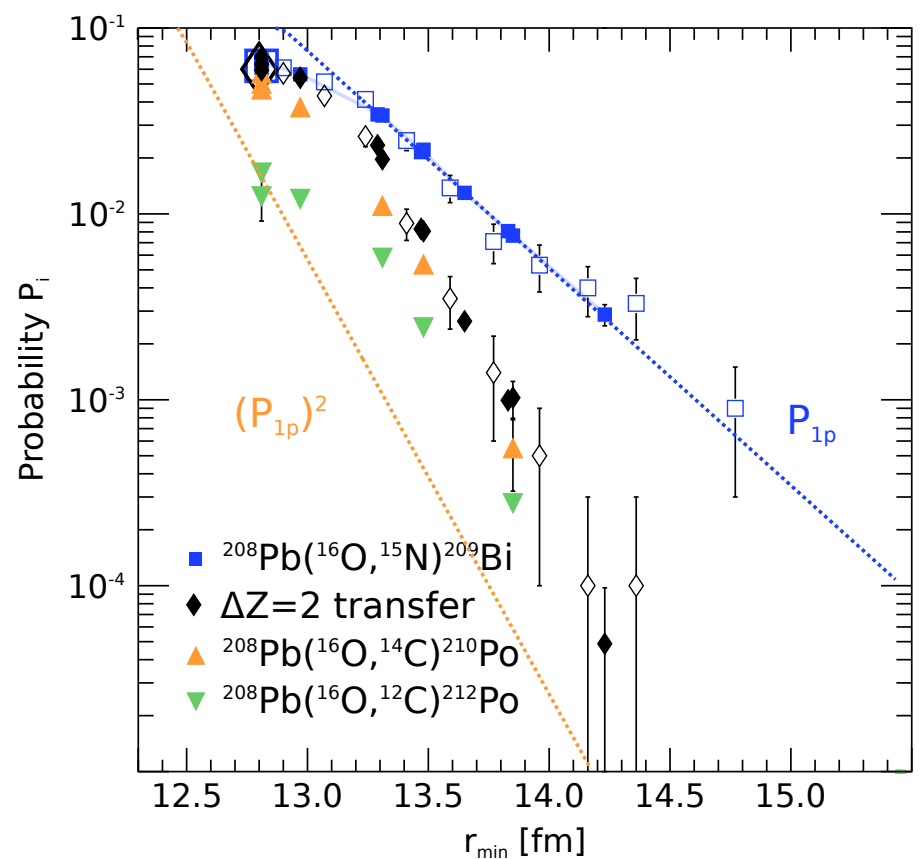

Figure 3: Transfer probabilities $P_{i}$ for the indicated transfer processes in the reaction ${ }^{16} \mathrm{O}+{ }^{208} \mathrm{~Pb}$ as a function of the distance of closest approach (see text). The asymptotic behaviour for $1 p$ transfer and predicted sequential $2 p$ transfer are shown by the dotted straight lines. The large open square and diamond (at $\left.r_{\min }=12.8 \mathrm{fm}\right)$ are the measurements for N (blue) and C PLFs (black) from Videbaek et al. [23]. The smaller open squares and diamonds are the measurements for N (blue) and C PLFs (black) from Timmers [24].

experiment, scattered from a thick tantalum target to give experimental energy loss curves, the accuracy of the calculated energy loss curves was confirmed. Details on the isotopic identification of the different PLFs can be found in Ref. [26].

For the reaction ${ }^{16} \mathrm{O}+{ }^{208} \mathrm{~Pb}$, the predominant contribution to the $\Delta Z=1$ events was identified with the $-1 p$ channel ( $1 p$-stripping: ${ }^{208} \mathrm{~Pb}\left({ }^{16} \mathrm{O},{ }^{15} \mathrm{~N}\right){ }^{209} \mathrm{Bi}$ ), and corresponding transfer probabilities are shown by the blue squares in Fig. 3, plotted as a function of the distance of closest approach $r_{\min }$ assuming trajectories in a Coulomb plus nuclear potential. Extracted transfer probabilities for the two dominant $\Delta Z=2$ channels $-2 p\left(2 p\right.$-stripping: $\left.{ }^{208} \mathrm{~Pb}\left({ }^{16} \mathrm{O},{ }^{14} \mathrm{C}\right){ }^{210} \mathrm{Po}\right)$ and $-2 p-2 n(\alpha$ particle stripping: $\left.{ }^{208} \mathrm{~Pb}\left({ }^{16} \mathrm{O},{ }^{12} \mathrm{C}\right){ }^{212} \mathrm{Po}\right)$ are shown in Fig. 3 by the orange and green triangles, respectively. Results show that at sub-barrier energies $2 p$ transfer is the dominant process, $\alpha$ particle transfer probabilities being smaller by a factor of $\sim 2-3$. The difference in probabilities between $2 p$ and $\alpha$ transfer increases with increasing beam energy, and is largest at $E_{c . m} / V_{B} \sim 1.0$.

For the reaction ${ }^{32} \mathrm{~S}+{ }^{208} \mathrm{~Pb}$, an equivalent analysis also indicates that $2 p$-stripping is the dominant $\Delta Z=2$ transfer process compared to $\alpha$-particle transfer. Resulting extracted transfer probabilities for $\Delta Z=1$ and $\Delta Z=2$ transfer are shown in Fig. 4. Due to the similarity in reaction $Q$-values of different transfer channels for both $\Delta Z=1$ and $\Delta Z=2$ processes, deduced transfer probabilities may contain contributions from the channels $-1 p$ and $-1 p+1 n$ for $\Delta Z=1$, and $-2 p-1 n$ and $-2 p+2 n$ for $\Delta Z=2$, respectively.

Overall the obtained absolute probabilities for transfer in both the ${ }^{16} \mathrm{O}+{ }^{208} \mathrm{~Pb}$ and ${ }^{32} \mathrm{~S}+{ }^{208} \mathrm{~Pb}$ reactions, show excellent agreement with previous transfer measurements of the $\Delta Z=1$ and $\Delta Z=2$ 


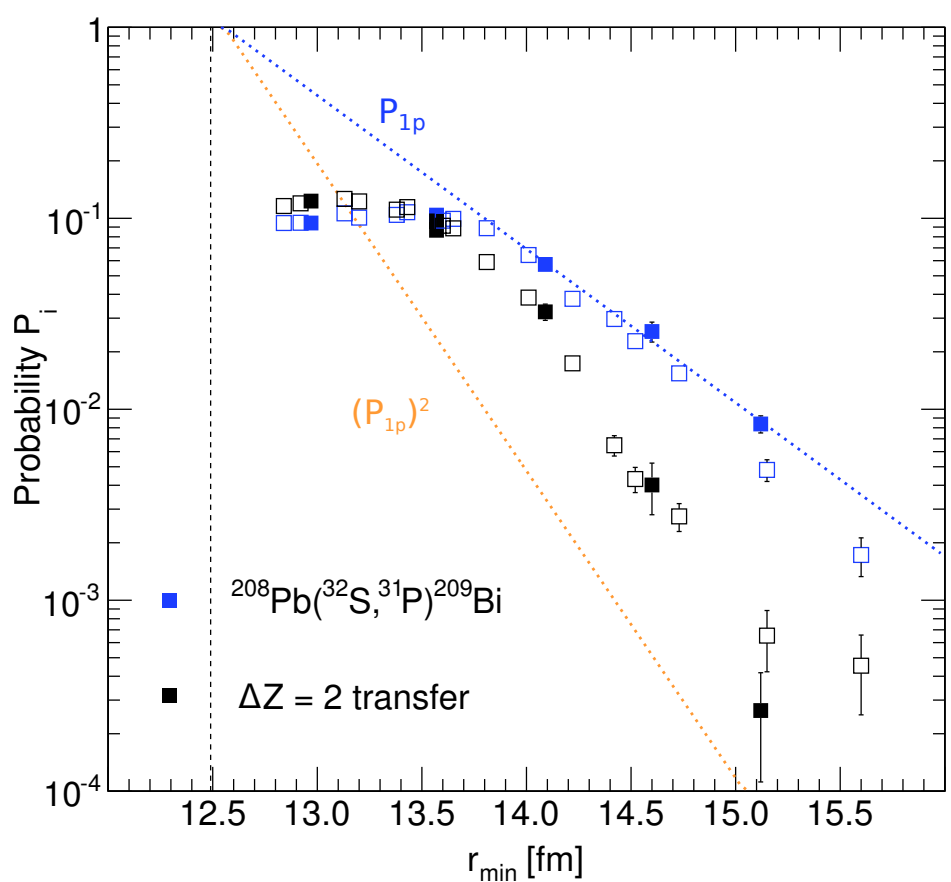

Figure 4: Transfer probabilities $P_{i}$ for the indicated transfer processes in the reaction ${ }^{32} \mathrm{~S}+{ }^{208} \mathrm{~Pb}$ as a function of the distance of closest approach $r_{\min }$ (see text). The asymptotic behaviour for $1 p$ transfer and predicted sequential $2 p$ transfer are shown by the dotted straight lines. The open squares are the measurements for $\mathrm{P}$ (blue) and Si (black) from Timmers [24].

transfer events from Refs. [23, 24], shown by the open square symbols in Figs. 3 and 4.

\section{Excitation energies}

The energies lost to excitation of the residual nuclei following $\Delta Z=1$ and $\Delta Z=2$ transfer were determined using the corresponding dominant transfer reactions leaving the residual nuclei in their ground states as the reference processes. Fig. 5 shows excitation energy spectra $d P / d E_{x}$ as a function of $E_{x}$ for energies corresponding to $E_{c . m} . / V_{B}=0.96,1.00,1.05$ for the PLFs following $\Delta Z=2$ transfer in the ${ }^{16} \mathrm{O}$ - (top panel) and ${ }^{32} \mathrm{~S}$-induced reactions (bottom panel). As can be seen from the figure, average excitation energies increase with increasing charge product of the colliding nuclei, associated with increasing matter overlap between target and projectile nucleus, see also Ref. [6, 20]. All reactions show the population of highly excited states in the target-like nuclei [26], even at beam energies well below the fusion barrier. At an energy $\sim 5 \mathrm{MeV}$ below the barrier, excitation energies up to $\sim 15 \mathrm{MeV}$ are observed for the ${ }^{16} \mathrm{O}$-induced reaction and $\sim 25 \mathrm{MeV}$ for the ${ }^{32} \mathrm{~S}$-induced reaction.

Since the level density of single-particle states in a nucleus $n$ scales exponentially with the square root of the excitation energy $n \propto \exp \sqrt{E_{x}}$, processes leading to large excitation energies indicate that the energy is dissipated amongst a very large number of single particle states. This irreversible loss of kinetic energy due to energy dissipation at sub-barrier energies is believed to be the key process responsible for the suppression of fusion at these energies, in a similar way as 

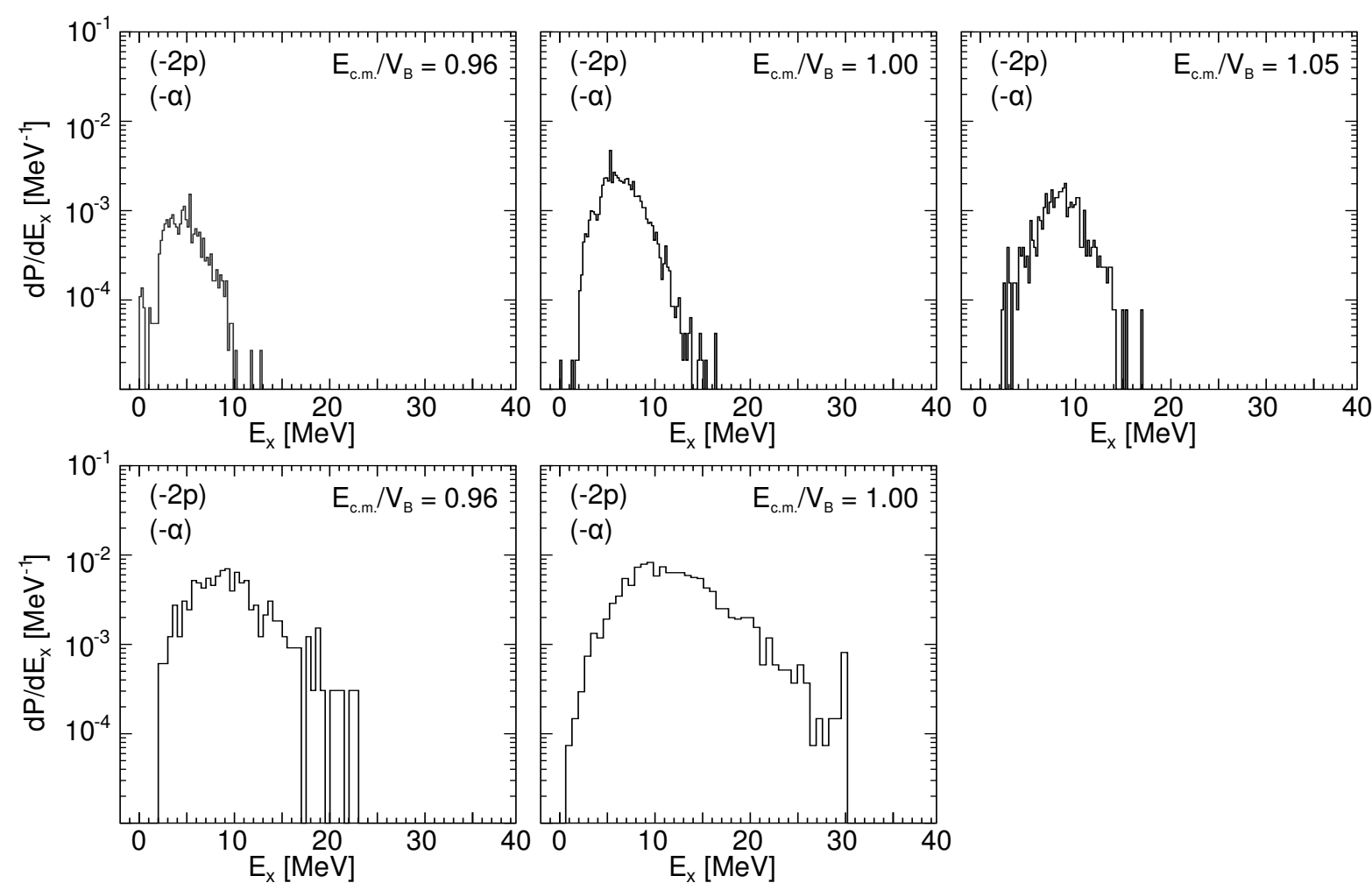

Figure 5: Excitation energy spectra showing the differential transfer probabilities $d P / d E_{x}$ as a function of the excitation energy $E_{x}$ (i.e. kinetic energy loss taking into account the specific reaction $Q$ value) of the PLFs at the indicated energies for the $\Delta Z=2$ transfer processes ( $2 p, \alpha$ transfer) in the reactions ${ }^{16} \mathrm{O}+{ }^{208} \mathrm{~Pb}$ (top panel) and ${ }^{32} \mathrm{~S}+{ }^{208} \mathrm{~Pb}$ (bottom panel).

energy dissipation through deep inelastic collisions has been linked to the suppression of fusion at above-barrier energies [27].

\section{Phenomenological dissipative coupled channels model}

A new phenomenological framework has been developed to investigate the effect of energy dissipation on the fusion probability for the reaction ${ }^{16} \mathrm{O}+{ }^{208} \mathrm{~Pb}$. In this approach the probability for fusion is reduced due to dissipative loss of kinetic energy into nucleonic degrees of freedom, which is directly linked to the measured probability for populating excited states with $E_{x} \geq E_{x, \text { thresh }}$ following transfer. The population of states below the threshold excitation energy $E_{x}<E_{x \text {,thresh }}$ are treated within the traditional coherent coupled channels framework. A diagrammatic illustration of the key components of this new model are shown in Fig. 6 for collisions involving heavy (top panel) and light nuclei (bottom panel).

Results of a calculation using both couplings to low-lying collective states in the ${ }^{208} \mathrm{~Pb}$ and dissipative energy loss due to high- $E_{x}$ transfer are shown in Fig. 7 by the solid red curves. Also shown in the figure by the dotted black curves are standard optical model (single-channel) calculations. All coupling and potential parameters were taken from a previous analysis of the total quasi-elastic scattering flux in the same reaction, as detailed in Refs. [2, 3]. A threshold energy of 


\section{heavy nucleus}

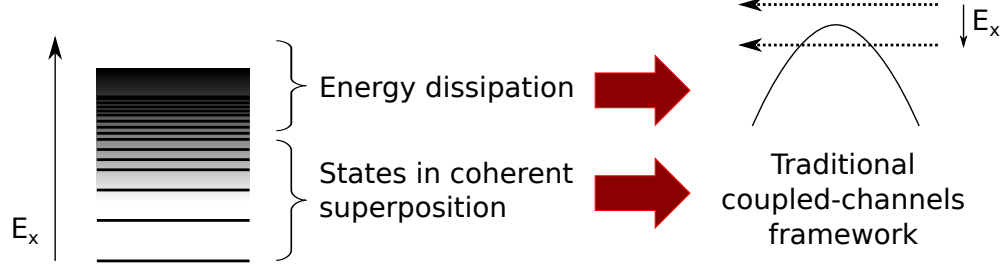

\section{light nucleus}

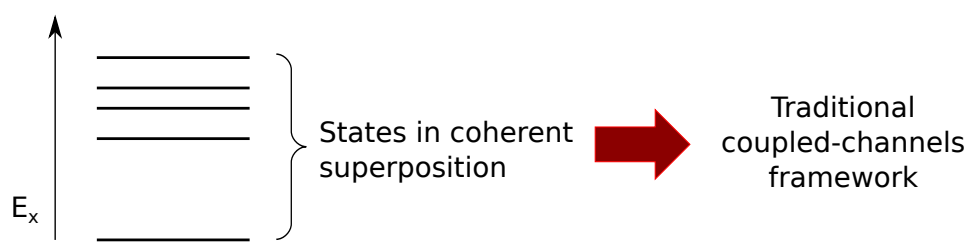

Figure 6: Top panel: The population of highly excited states following nucleon transfer in heavy nuclei leads to irreversible loss of kinetic energy into the many excited states, that can be considered to act like a heat bath. This in turn leads to a reduction of the probability for fusion. The population of low-lying excited states is treated within the traditional coherent coupled reaction channels framework. Bottom panel: In light nuclei the level density of single particle states at excitation energies typical for nuclear collisions at subbarrier energies is small. Population of these distinct states is treated within the coherent coupled reaction channels framework and does not lead to irreversible dissipation of kinetic energy.

$E_{x, \text { thresh }}=8.0 \mathrm{MeV}$ was used, which was taken to mark the onset of energy dissipation through the population of excited states in the nuclei. The left panel in Fig. 7 shows the fusion cross sections, the right panel the fusion barrier distribution [28] $B_{\text {fus }}=d^{2}\left(E_{c m} \sigma_{\text {fus }}\right) / d E_{c m}^{2}$. The agreement of the measured fusion cross sections with the calculations where energy dissipation was included is excellent. Agreement with the fusion barrier distribution is also good, in particular the position of the average barrier height at $E_{c m}=74.5 \mathrm{MeV}$ is correctly reproduced.

\section{Conclusion}

In conclusion, the following results were obtained from a detailed analysis of the projectilelike fragments detected at a backward angle in the reactions ${ }^{16} \mathrm{O},{ }^{32} \mathrm{~S}+{ }^{208} \mathrm{~Pb}$ :

1. Transfer of one and two protons ( $1 p$ and $2 p$-stripping) in the reactions ${ }^{16} \mathrm{O},{ }^{32} \mathrm{~S}+{ }^{208} \mathrm{~Pb}$ occurs with a significant probability already at energies well below the fusion barrier. Transfer of $2 p$ is the predominant $\Delta Z=2$ transfer process, with absolute probabilities being $\sim 2-3$ times larger than those for $\alpha$-particle transfer.

2. The (heavy) target-like residual nuclei following $2 p$ and $\alpha$-particle transfer are left in highly excited states, with excitation energies up to $\sim 15 \mathrm{MeV}$ and $\sim 25 \mathrm{MeV}$ for the ${ }^{16} \mathrm{O}$ - and ${ }^{32} \mathrm{~S}$-induced reactions, respectively.

A new phenomenological and probabilistic approach to calculate fusion cross sections taking into account energy dissipation has been presented. In this approach the irreversible loss of kinetic 

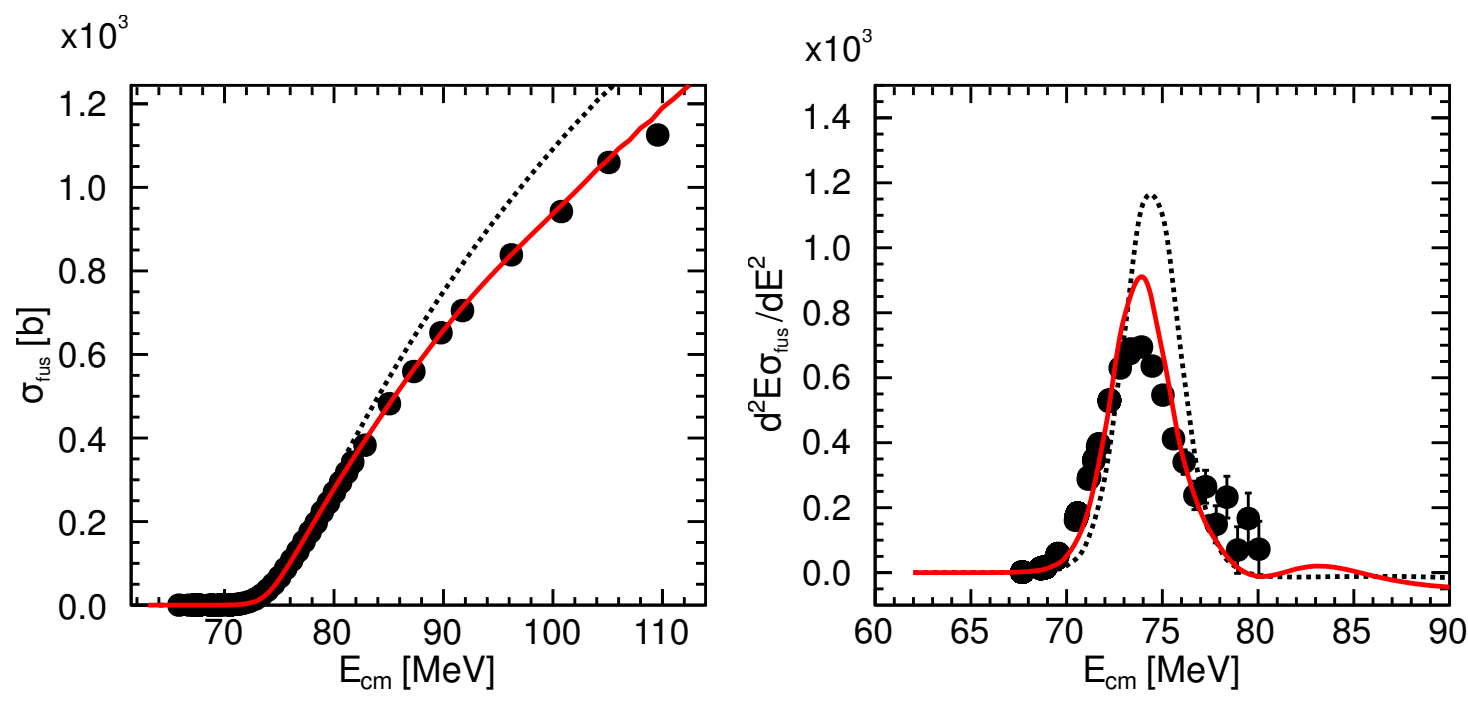

Figure 7: Fusion cross sections and barrier distribution for the reaction ${ }^{16} \mathrm{O}+{ }^{208} \mathrm{~Pb}$. Experimental data are from Ref. [27]. The dotted black curve corresponds to a simple optical model calculation. The solid red curve corresponds to a coupled channels calculations including energy dissipation to nucleonic degrees of freedom, see text. Coupling and potential parameters were taken from Ref. [2]

energy due to the population of highly excited states following transfer leads to a suppression of the fusion probability at energies above the fusion barrier. Transfer probabilities for populating different excited states in the residual nuclei were directly obtained from the measured $1 p$ and $2 p$ transfer probabilities in the reaction ${ }^{16} \mathrm{O}+{ }^{208} \mathrm{~Pb}$. Results using the traditional coherent coupledchannels model for reaction channels populating states with excitation energies $E_{x}<8.0 \mathrm{MeV}$ and a phenomenological approach to include irreversible dissipation of kinetic energy due to the population of states with $E_{x}>8.0 \mathrm{MeV}$, show that excellent agreement with measured fusion cross sections at energies above the fusion barrier can be obtained. Calculations also give good agreement with the measured fusion barrier distribution.

\section{Outlook}

Results presented in this work strongly support the idea that few-nucleon transfer triggers the onset of dissipative and irreversible processes in the collision of nuclei already at energies well-below the fusion barrier. This may reduce the tunnelling probability, and suppress the fusion yield at these energies in a similar way as was demonstrated within the new empirical framework for the reaction ${ }^{16} \mathrm{O}+{ }^{208} \mathrm{~Pb}$ at above-barrier energies. With this suggested link between deep subbarrier fusion hindrance and the population of a large number of excited states in the residual nuclei following transfer, deep sub-barrier fusion hindrance in lighter systems such as ${ }^{12} \mathrm{C}+{ }^{12} \mathrm{C}$ might play only a negligible role, since level densities of single particle states at excitation energies typical for collisions at sub-barrier energies are much smaller in lighter nuclei. This might have a significant affect on the extrapolation of $S$-factors for reactions involving light nuclei down to energies relevant to different astrophysical scenarios. 


\section{References}

[1] M. Dasgupta et al., Annu. Rev. Nucl. Part. Sci. 48, 401 (1998).

[2] M. Evers et al., Phys. Rev. C 78, 034614 (2008).

[3] M. Evers et al., Phys. Rev. C 81, 014602 (2010).

[4] C. L. Jiang et al., Phys. Rev. Lett. 89, 052701 (2002).

[5] C. L. Jiang et al., Phys. Rev. C 71, 044613 (2005).

[6] M. Dasgupta et al., Phys. Rev. Lett. 99, 192701 (2007).

[7] H. Esbensen et al., Phys. Rev. C 76, 054609 (2007).

[8] M. High et al., Nucl. Phys. A 282, 181 (1977).

[9] J. Patterson et al., Astrophys. J. 157, 367 (1969).

[10] M. G. Mazarakis et al., Phys. Rev. C 7, 1280 (1973).

[11] H. Becker et al., Z. Phys. A 303, 305 (1981).

[12] L. Barrón-Palos et al., Nucl. Phys. A 779, 318 (2006).

[13] T. Spillane et al., Phys. Rev. Lett. 98, 122501 (2007).

[14] M. L. Chatterjee et al., Nucl. Phys. A 333, 273 (1980).

[15] C. L. Jiang et al., Phys. Rev. C 75, 015803 (2007).

[16] C. L. Jiang et al., Phys. Rev. C 79, 044601 (2009).

[17] L. R. Gasques et al., Phys. Rev. C 76, 035802 (2007).

[18] L. R. Gasques et al., Phys. Rev. C 76, 024612 (2007).

[19] C. L. Jiang et al., Phys. Rev. C 75, 057604 (2007).

[20] J. O. Newton et al., Phys. Rev. C 70, 024605 (2004).

[21] C. H. Dasso et al., Phys. Rev. C 39, 2073 (1989).

[22] V. Zagrebaev et al., J. Phys. G: Nucl. Part. Phys. 34, 1 (2007).

[23] F. Videbaek et al., Phys. Rev. C 15, 954 (1977).

[24] H. Timmers, Ph.D. thesis, The Australian National University (1996).

[25] J. F. Ziegler et al., The Stopping and Range of Ions in Solids, volume 1 of The Stopping and Ranges of Ions in Matter, Pergamon Press (1985).

[26] M. Evers et al., Phys. Rev. C 84, 054614 (2011).

[27] C. R. Morton et al., Phys. Rev. C 60, 044608 (1999).

[28] N. Rowley et al., Phys. Lett. B 254, 25 (1991). 